with the smaller real part can be developed by the method of successive approximations exactly as the solution corresponding to the exponent with the larger real part is developed in my article in the Transactions, provided that $R x<1-k$, where $x$ is the difference of the exponents at $x=0$ so taken that $R x>0$.

By using this theorem we deduce the

Second Theorem of Comparison: If the conditions of the first theorem of comparison are fulfilled, and if moreover both equations have the same exponents, and both satisfy restriction (A); and if $\bar{y}_{1}$ and $\bar{y}_{2}$ are the solutions corresponding to the smaller exponent; and if $\bar{y}_{1}$ vanishes when $x=\bar{x}_{1}>0$, but not in the interval $0<x<\overline{x_{1}}$, then $\overline{y_{2}}$ will vanish at least once in this interval provided that $x<1-k$.

Finally I should like to mention a fact which had escaped my notice until after my paper in the Transactions was printed, namely that the class of singular points which I there discuss under the name regular can be brought into very close connection with the class of singular points previously studied by Kneser ( Crelle's Journal, Volumes 116,117, 120 ; Mathematische Annalen, Volume 49). This can be done by replacing the independent variable $x$, which I use, by $z$ where $x=e^{-z}$. Although many of my results can be deduced by this method from those previously found by Kneser and vice versa, the results in the two cases are by no means coextensive, nor does either include the other. I shall come back to this matter more at length on a subsequent occasion. It may be noted that the method of successive approximations can also be applied directly to Kneser's case.

Harvard University, Cambridge, Mass.

\title{
NOTE ON THE ENUMERATION OF THE ROOTS OF THE HYPERGEOMETRIC SERIES BETWEEN ZERO AND ONE.
}

\author{
BY DR. M. B. PORTER.
}

(Read before the American Mathematical Society, February 24, 1900.)

In the May number of the Bulletin for 1897, the writer gave a solution of the problem of enumerating the real roots of $F(\alpha, \beta, \gamma, x)$ between zero and one which depended on two well known theorems of Sturm-there referred to as [A] 
and $[\mathrm{B}] *$-and Gauss' expression of $F(\alpha, \beta, \gamma, x)$ in terms of the two linearly independent solutions of the hypergeometric equation about the point $x=1$. The investigation there given can be considerably simplified and Gauss' formula avoided by the use of the following theorem [C]: $\dagger$ If $S(x)=\sum_{0}^{\infty} a_{n} x^{n}$ is divergent for $x=r$ but convergent for $|x|<|r|$, and if $S(x)$ diverge to $+\infty(-\infty)$ as $x$ approaches $r$, then $\lim _{n=\infty} S_{n}(r)=+\infty(-\infty)$ where $S_{n}(r)=\sum_{0}^{n} a_{n} r^{n}$.

In the paper above mentioned it is shown that the problem of determining the number $X$ of roots of the solution of the hypergeometric equation corresponding to the larger exponent of $x=0$ can be reduced to the case where $\lambda, \mu$. and $\nu \neq$ (the exponent differences of $0, \infty$, and 1 respectively) are not less than zero.

To determine $X$ in this case it is only necessary to determine the number of roots lost by $F(\alpha, \beta, \gamma, x)$ when $\beta$, by the decrease of $\mu$, increases from some initial negative value to zero.

By the first theorem of Sturm quoted, $F(\alpha, \beta, \gamma, x)$ is losing or certainly not gaining roots when, under the conditions laid down, $\mu$ decreases.

Moreover since $\nu \geq 0, F(\alpha, \beta, \gamma, 1)$ is divergent; and by [C] $F(\alpha, \beta, \gamma, 1-0)$ changes from + to $-(-$ to +$)$ when $\beta$ increases through an odd (even) negative integer.

But when $F(\alpha, \beta, \gamma, 1-0)$ changes sign, $\beta$ increasing (i. e., $\mu$ decreasing), an odd number of roots must have been lost by $F(\alpha, \beta, \gamma, x)$ in the interval $0-1$ and it is easily seen (by considering the polynomials obtained when $\beta$ is integral) that exactly one root is lost. Thus when $\lambda, \mu$, and $\nu$ are not less than zero,

$$
X=E(1-\beta) \text {. }
$$

In the same way it may be shown that in any case

$$
X=E\left(\frac{\mu-|\lambda|-|\nu|+1}{2}\right) \text {. }
$$

When $\lambda<0$, the number $N$ of roots of $F(\alpha, \beta, \gamma, x)$

* Theorem [B] is a slightly generalized form of the theorem given by Sturm, Liouville's Journal, vol. I.

† Cf. Stolz, Allgemeine Arithmetik, p. 279.

$\ddagger \lambda=\gamma-1, \mu=\alpha-\beta, v=\alpha+\beta-\gamma$.

$\& E(n)$ is used to denote the largest positive integer less than $n$, so that $E(n)=0$ if $n \leqq 1$. 
between 0 and 1 is by $[B]$ either $X$ or $X+1$, the even or odd value of $N$ being chosen according as $F(\alpha, \beta, \gamma, 1-0)$ is greater or less than 0 , viz., $1^{\circ}$ If $\nu>0$, according as $E(-\alpha)+E(-\beta)+E(-\gamma)$ is odd or even ; $2^{\circ}$ if $\nu<0$, according as $E(\alpha-\gamma)+E(\beta-\gamma)+E(-\gamma)$ is odd or even. If, however, in $1^{\circ}$ either $-\alpha$ or $-\beta$ is integral and positive $F(\alpha, \beta, \gamma, x)$ ceases to be linearly independent of the solution corresponding to the larger exponent of $x=1$, so that, by $[B], N=X$. Similarly in $2^{\circ}$, if $\alpha-\gamma$ or $\beta-\gamma$ is a positive integer, $N=X$ for the same reason.

YALE UNIVERSITY, February 12, 1900 .

\section{THE SUMMER MEETING OF THE DEUTSCHE MATHEMATIKER-VEREINIGUNG AT MUNICH, SEPTEMBER, 1899.}

Probably no mathematical society in the world brings together at its annual meetings so many illustrious mathematicians and exhibits an activity of such multifarious nature and far-reaching importance as the Deutsche Mathematiker-Vereinigung. Founded about a decade ago through the efforts of G. Cantor, W. Dyck and others, it has enjoyed the utmost prosperity. It is indeed to be regretted that its meetings are held so late in the summer that it is impossible, save in exceptional circumstances, for our own members to be present. Such a piece of good fortune befell me last year, and I hope the following personal reminiscences may prove interesting enough to justify their publication. For more details of scientific character I refer to the Chronik of the Vereinigung, the article by E. Lampe in the Naturwissenschaftliche Rundschau, (1899), p. 537, and the official Verhandlungen der Gesellschaft Deutscher Naturforscher und Aertze, Vogel, Leipzig, 1900.

The summer meetings of the Vereinigung are always held in connection with those of the Gesellschaft Deutscher Naturforscher und Aertze, which corresponds in Germany to our American Association for the Advancement of Science. This year the meeting was held September 17th to $23 \mathrm{~d}$ at Munich, a city justly celebrated for its scientific institutions and collections of art, for its beautiful surroundings, and for the good nature and kindly spirit of its population. 